\title{
Transient Hemi-Lower Limb Ischemia in the Newborn: Arterial Thrombosis or Persistent Sciatic Artery?
}

\author{
Makiko Kirino, $\mathrm{MD}^{1}$ Masayuki Ochiai, MD, $\mathrm{PhD}^{1}$ Masako Ichiyama, $\mathrm{MD}^{1,2}$ Hirosuke Inoue, MD, $\mathrm{PhD}^{1}$ \\ Takeshi Kusuda, MD, $\mathrm{PhD}^{1,3}$ Tadamune Kinjo, MD, $\mathrm{PhD}^{1,4}$ Masataka Ishimura, MD, $\mathrm{PhD}^{1}$ \\ Shouichi Ohga, MD, $\mathrm{PhD}^{3,5}$
}

${ }^{1}$ Department of Pediatrics, Graduate School of Medical Sciences, Kyushu University, Fukuoka, Japan

2 Department of Pediatrics, Fukuoka Children's Hospital and Medical Center, Fukuoka, Japan

${ }^{3}$ Department of Pediatrics, Faculty of Medicine and Health Sciences, Yamaguchi University, Ube, Japan

${ }^{4}$ Department of Pediatrics, University of Occupational and Environmental Health, Kitakyushu, Japan

${ }^{5}$ Department of Perinatal and Pediatric Medicine, Graduate School of Medical Sciences, Kyushu University, Fukuoka, Japan
Address for correspondence Masayuki Ochiai, MD, PhD, Department of Pediatrics, Graduate School of Medical Sciences, Kyushu University, 311 Maidashi, Higashi-ku, Fukuoka 812-8582, Japan (e-mail: ochimasa@pediatr.med.kyushu-u.ac.jp).

\begin{abstract}
Keywords

- thromboembolism

- vascular anomaly

- heparin

- antithrombin

Neonatal thromboembolism occurs with various predispositions and triggers. Early diagnosis of the thrombosis is challenging and essential for the therapeutic interventions. We herein report two newborns who presented with transient hemi-lower limb ischemia due to (1) arterial thrombosis or (2) a persistent sciatic artery (PSA). The patient with arterial thrombosis showed elevations of fibrin degradation product and Ddimer and received antithrombin and heparin intravenously. The patient with PSA was immediately assessed by a contrast-enhanced computed tomography because of a transient ischemic episode with no evidence of hypercoagulability. Newborns suspected of having arterial thrombosis may need urgent surgical intervention along with thrombolytic and anticoagulant therapy to prevent organ ischemia and amputation of extremities. Conversely, some PSA cases have reportedly been treated conservatively. This vascular anomaly was previously reported as a cause of lower limb ischemia only in a newborn. PSA is a critical differential diagnosis of neonatal arterial thrombosis that needs urgent therapeutic intervention.
\end{abstract}

Thromboembolism is a multifactorial disease involving genetic predispositions, underlying disorders, and acquired triggers. The established genetic risks for thrombosis beyond the race/ethnicity include protein $C(P C)$, protein $\mathrm{S}$ (PS), and antithrombin (AT) deficiencies. ${ }^{1}$ PC deficiency is a major critical thrombophilia of the newborns who presents with purpura fulminans, intracranial thromboembolism, ${ }^{2}$ and fetal hydrocephalous. ${ }^{3}$ Newborn infants are at the highest risk of thrombosis throughout childhood even without having the inherited thrombophilias. ${ }^{4,5}$ The incidence of embolism has been increasingly diagnosed in infants and children, as a complication of sepsis, cancer, congenital heart disease, therapy-related events of drugs, and intravenous catheters. ${ }^{6}$ Recent advances in the neonatal medicine may raise the chance of developing thromboembolism. ${ }^{7}$ received

July 11,2016

accepted after revision

December 12, 2016
DOI http://dx.doi.org/

10.1055/s-0037-1598044. ISSN 2157-6998.
Copyright $\odot 2017$ by Thieme Medical Publishers, Inc., 333 Seventh Avenue, New York, NY 10001, USA. Tel: +1(212) 584-4662.

\section{License terms \\ (요 $\Theta \circledast$}


Early diagnosis of thromboembolism is challenging in the newborn because of the physiological increase of the natural anticoagulants and the difficulty of the imaging analysis to determine the vascular occlusions, although an outline of the management has been proposed. ${ }^{8}$ Another concern is the limited use of the expensive PC agents for replacement therapy. ${ }^{2}$ We herein report two newborns who presented with transient hemi-lower limb ischemia due to (1) arterial thrombosis or (2) a persistent sciatic artery (PSA). Newborns suspected as having arterial thrombosis may need urgent surgical intervention along with thrombolytic and anticoagulant therapy to prevent organ ischemia and amputation of extremities. Conversely, some of PSA cases were reported to be treated conservatively. It is therefore essential to diagnose the etiology of neonatal lower limb ischemia promptly for proper therapeutic intervention.

\section{Case Presentations}

Patient 1: A Female Preterm Infant Presenting with Transient Ischemia of the Left Lower Abdominal Wall and Limb Due to Arterial Thrombosis

A female infant was born at 34 weeks of gestation through an induced delivery from a multiparous mother. The pregnant course had been complicated with pregnancy induced hypertension, and the family history did not reveal any thrombotic and ischemic diseases. The female infant was an appropriate-for-gestational age (AGA) phenotype and the birth weight was $1,945 \mathrm{~g}$. The Apgar scores were 9 and 9 points at 1 and 5 minutes, respectively. The umbilical cord and placenta did not demonstrate either ischemic or thrombotic lesions.

After birth, we noted the infant's left lower abdominal wall and limb to be quite pale on the initial evaluation (-Fig. 1A). No pulsations were detected in the left femoral, popliteal, and posterior tibial arteries. Fifteen minutes after the evaluation, the circulatory disturbance of the affected side was spontaneously recovered. The initial laboratory examination revealed elevations of fibrin degradation product (FDP; (88.8; reference range [rr]: $<5 \mu \mathrm{g} / \mathrm{mL}$ ) and D-dimer ( $40.7 ; \mathrm{rr}:<1.0 \mu \mathrm{g} / \mathrm{mL})$. We therefore speculated the occurrence of arterial thrombosis as a possible etiology of the ischemia and therefore administered AT (60 IU/kg/day) and unfractionated heparin (10 IU/kg/hour) intravenously to the patient. However, we were unable to detect any thrombotic lesions in the affected arteries on an ultrasonographic examination. The prothrombotic state thereafter gradually improved, and the administration of both AT concentrate and heparin was completed on the 2nd (FDP: 7.9 $\mu \mathrm{g} / \mathrm{mL}$; and D-dimer: $5.1 \mu \mathrm{g} / \mathrm{mL}$ ) and 16th day of life (FDP: $<2.6$ $\mu \mathrm{g} / \mathrm{mL}$; D-dimer: $1.1 \mu \mathrm{g} / \mathrm{mL}$ ), respectively. A slight decline in the platelet counts was observed soon after the treatment $\left(234 \times 10^{9}, 221 \times 10^{9}\right.$, and $543 \times 10^{9}$ on the $1 \mathrm{st}, 2^{\text {nd }}$, and 16 th day, respectively). At the time the infant was discharged from the hospital, neither an ultrasonographic examination nor magnetic resonance imaging (MRI) revealed any thromboembolic lesions in the cardiovascular and central nervous system. No inherited deficiency of PC, PS, and AT was determined.

\section{Patient 2: A Male Preterm Infant Presenting with Transient Ischemia of the Left Lower Limb Due to a Persistent Sciatic Artery}

A male infant was born at 34 weeks' gestation through an induced vaginal delivery from a nulliparous mother. The pregnant course had been complicated with a preterm-premature rupture of the membrane. The family history did not reveal any history of either thrombotic or ischemic diseases. The boy was an AGA phenotype and the birth weight was $1,930 \mathrm{~g}$. The Apgar scores were 9 and 9 points at 1 and 5 minutes, respectively. The umbilical cord and placenta both demonstrated normal findings.

After birth, we noted his left lower abdominal wall and limb to be quite pale on the initial evaluation ( $\mathbf{F i g . ~ 1 B )}$. Pulsations of the left femoral, popliteal, and posterior tibial arteries were diminished compared with the right ones. However, the patient was able to move the affected extremity spontaneously and did not appear to be in any

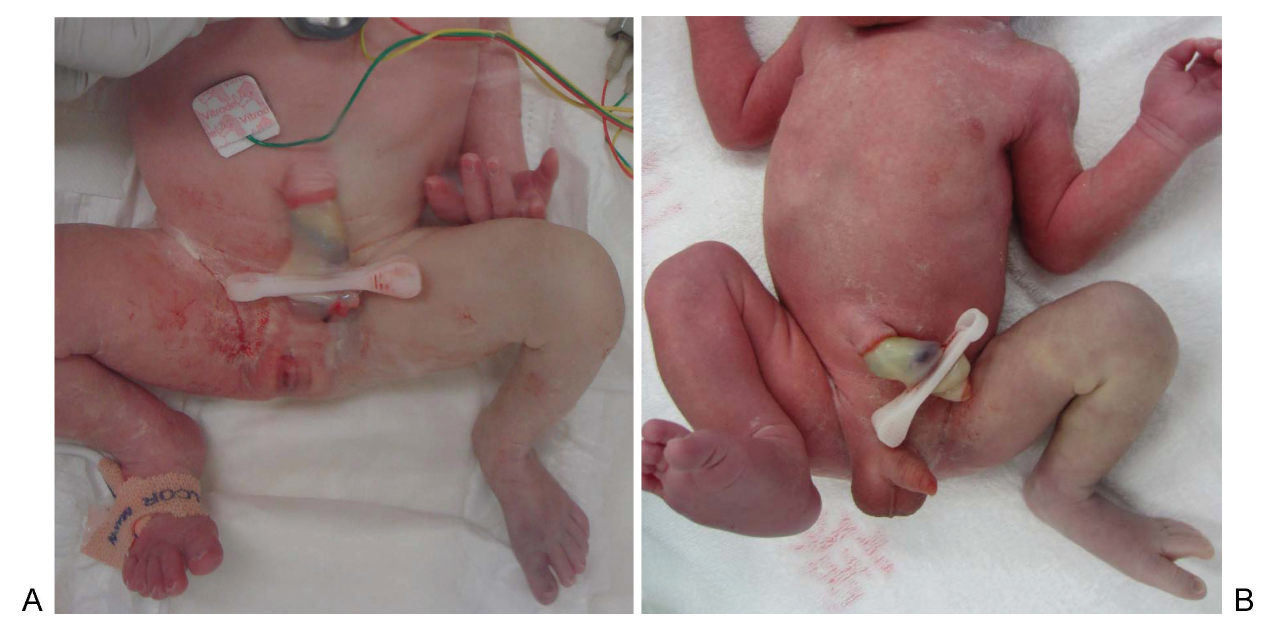

Fig. 1 Neonates complicated with circulatory disturbances of the hemi-lower limb. A transient ischemia of the left lower abdominal wall and limb due to arterial thrombosis (A) and left lower limb due to a persistent sciatic artery (B). 

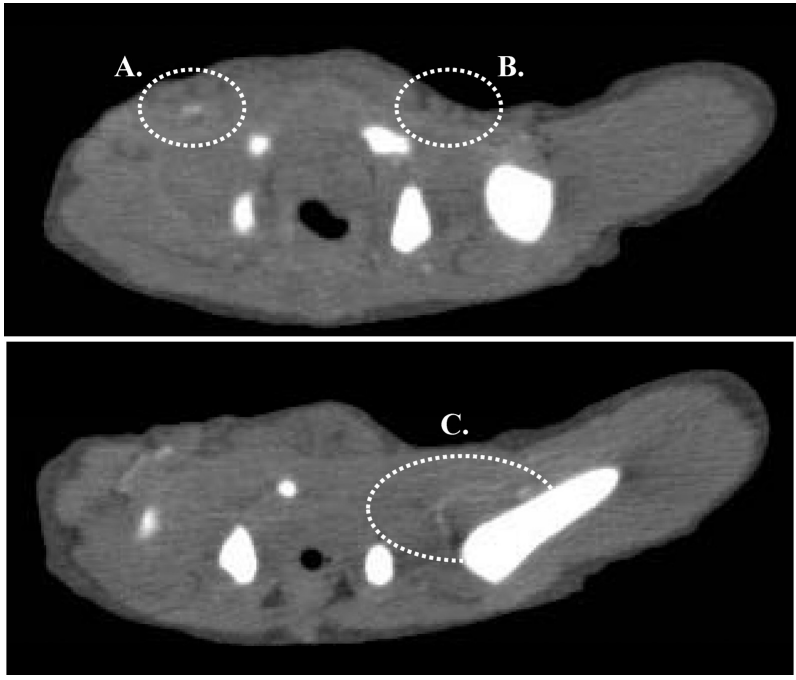

Fig. 2 A contrast-enhanced computed tomography finding of the persistent sciatic artery (PSA). The left external iliac artery and the femoral artery (A) were diminished compared with the right (B). The superficial and profunda femoral artery reconstructed collateral vessels of the internal and PSA (C).

obvious pain or distress. An emergency ultrasonographic examination did not detect any thrombotic lesions in the affected common femoral artery. Initial laboratory tests showed a slight elevation of FDP $(12.0 ; \mathrm{rr}:<5 \mu \mathrm{g} / \mathrm{mL})$ and Ddimer (6.3; rr: $<1.0 \mu \mathrm{g} / \mathrm{mL})$. Contrast-enhanced computed tomography (CT) performed 6 hours after birth revealed the left external iliac artery and the femoral artery ( - Fig. 2A) to be diminished in comparison to the right (-Fig. 2B). The superficial and profunda femoral artery also reconstructed collateral vessels of the internal and persistent sciatic artery (-Fig. 2C). Based on the laboratory and image findings, the patient was diagnosed as having transient ischemia due to external compression of the collateral vessels, but not arterial thrombosis. Twelve hours after birth, the peripheral circulation of the affected limb gradually improved. Thereafter, no recurrent episodes of ischemic limbs were observed.

\section{Discussion}

\section{Arterial Thrombosis Is a Frequently Encountered Complication in the Neonatal Intensive Care}

Arterial thrombosis is one of the frequently demonstrated complications in the newborn infants treated in the neonatal intensive care units. ${ }^{9}$ Klinger et al $^{10}$ reviewed 63 cases of severe aortic thrombosis in term and preterm infants. The available laboratory data revealed an elevation in the serum creatinine level (84\%) and plasma D-dimer level (50\%), a decline in the plasma fibrinogen level (57\%) and platelet count (51\%), a prolonged prothrombin time (PT), or an activated partial thromboplastin time (APTT; 47\%). We previously experienced a case of fetal ventriculomegaly and neonatal stroke associated with a PC deficiency. ${ }^{3}$ Saxonhouse ${ }^{11}$ and Will ${ }^{8}$ published the guidelines on the management of neonatal thrombosis. The primary goal of early intervention for the neonatal thrombosis in those guidelines was to prevent to prevent organ ischemia and amputation of extremities. Unfortunately, few randomized clinical trials have addressed the management of neonatal thromboembolism emergencies. A prompt diagnosis is quite essential to achieve an improvement in such patients, and the successful therapeutic strategies have been reported to be the intravenous administration of high-dose urokinase, ${ }^{12}$ low-molecular-weight heparin, ${ }^{10}$ and the combination of unfractionated heparin and AT concentrate. ${ }^{13}$ Expectant management is a reasonable alternative considering that recommendations and dosing regimens for anticoagulant/thrombolytic therapy in neonates are based on those reports. Meanwhile, the potential for serious complications (intracranial hemorrhage) must be considered in any neonate before initiating antithrombotic therapy. ${ }^{11}$

\section{Persistent Sciatic Artery Could Represent Lower Extremity Ischemia without Hypercoagulability}

A PSA is a vascular anomaly that was first reported in a postmortem case. ${ }^{14}$ Van Hooft et al $^{15}$ reviewed the clinical presentation and outcome of 122 patients with PSA. The most effective diagnostic modalities were angiography, CT, MRI, and ultrasonography. The choice of treatment depends on the symptoms and the classification of PSA. Some cases were treated conservatively with anticoagulants. Shah et $\mathrm{al}^{16}$ reported a PSA

Table 1 Infants exhibiting ischemic lower limbs immediately after birth due to arterial thrombosis or vascular anomaly

\begin{tabular}{|c|c|c|c|c|c|c|c|c|c|}
\hline No. & Diagnosis & Risk factor & Plt & Fib. & PT/APTT & D-dimer & $\begin{array}{l}\text { Diagnostic } \\
\text { imaging }\end{array}$ & Treatment & Reference \\
\hline 1 & Aortic & UAC (70\%) & $51 \% \downarrow$ & $57 \% \downarrow$ & $47 \% \uparrow$ & $50 \% \uparrow$ & US, etc. & Anticoagulants & Klinger et al $^{10}$ \\
\hline & thrombosis & Asphyxia (19\%) & & & & & & & \\
\hline & (66 patients) & Sepsis (16\%) & & & & & & & \\
\hline 2 & $\begin{array}{l}\text { Arterial } \\
\text { thrombosis }\end{array}$ & $\mathrm{PIH}$ & 234 & 141 & $16.7 / 63.9$ & $40.7 \uparrow$ & US & $\begin{array}{l}\text { Heparin, } \\
\text { antithrombin }\end{array}$ & Patient 1 \\
\hline 3 & PSA & Unidentified & normal & normal & normal & n.d. & US, CT & Conservatively & Shah et al ${ }^{16}$ \\
\hline 4 & PSA & Unidentified & 161 & 154 & $15.1 / 52.6$ & 6.3 & US, CT & Conservatively & Patient 2 \\
\hline
\end{tabular}

Abbreviations: APTT, activated partial thromboplastin time (s); CT, computed tomography; D-dimer (ng/mL); Fib., fibrinogen (mg/dL); n.d., not described; PIH, pregnancy induced hypertension; PIt, platelet ( $\left.10^{3} / \mu \mathrm{L}\right)$; PSA, persistent sciatic artery; PT, prothrombin time (s); UAC, umbilical arterial catheter; US, ultrasonography. 
case of a newborn infant who presented after birth with an atrophic right lower extremity and ischemia. The initial laboratory studies did not reveal any abnormal findings regarding the complete blood count, PT-international normalized ratio (INR), APTT, and fibrinogen. The symptoms gradually improved over the next 4 days without any particular treatment.

\section{Conclusions}

We reviewed the infants who exhibited ischemia of the lower limbs immediately after birth ( - Table 1 ). Newborns suspected of having arterial thrombosis may need a prompt diagnosis and proper intervention to prevent organ ischemia and amputation of extremities. The possibility of PSA should thus be considered in the newborn infants who present with a circulatory disturbance of either the hemi- or bilateral lower limb, but no evidence of hypercoagulability assessed by the blood tests. Further accumulation of neonatal PSA cases would provide useful information for the adequate management of symptomatic vascular anomalies.

\section{Statement of Ethics}

The Institutional Review Board approved this investigation. Written informed consent was obtained from the caretakers for publication of this case report and accompanying images.

\section{Acknowledgments}

This study was supported in part by a grant from the KAKEN (\#15K09717 [M. O.] and \#26860809 [H.I.]). The authors have no financial relationship relevant to this article to disclose.

\section{References}

1 Ichiyama $\mathrm{M}$, Ohga $\mathrm{S}$, Ochiai $\mathrm{M}$, et al. Age-specific onset and distribution of the natural anticoagulant deficiency in pediatric thromboembolism. Pediatr Res 2016;79(01/01):81-86
2 Ohga S, Kang D, Kinjo T, et al. Paediatric presentation and outcome of congenital protein C deficiency in Japan. Haemophilia 2013; 19(03):378-384

3 Ichiyama M, Ohga S, Ochiai M, et al. Fetal hydrocephalus and neonatal stroke as the first presentation of protein $C$ deficiency. Brain Dev 2016;38(02):253-256

4 Matsunaga Y, Ohga S, Kinjo T, et al. Neonatal asphyxia and renal failure as the presentation of non-inherited protein C deficiency. J Perinatol 2013;33(03):239-241

5 Matsuoka W, Yamamura K, Uike K, Nagata H, Ohga S, Hara T. Tachyarrhythmia-induced cerebral sinovenous thrombosis in a neonate without cardiac malformation. Pediatr Neonatol 2014; 55(05):412-413

6 Tolbert J, Carpenter SL. Common acquired causes of thrombosis in children. Curr Probl Pediatr Adolesc Health Care 2013;43(07): 169-177

7 Nowak-Göttl U, Janssen V, Manner D, Kenet G. Venous thromboembolism in neonates and children-update 2013. Thromb Res 2013;131(1, Suppl 1):S39-S41

8 Will A. Neonatal haemostasis and the management of neonatal thrombosis. Br J Haematol 2015;169(03):324-332

9 Saxonhouse MA. Thrombosis in the neonatal intensive care unit. Clin Perinatol 2015;42(03):651-673

10 Klinger G, Hellmann J, Daneman A. Severe aortic thrombosis in the neonate-successful treatment with low-molecular-weight heparin: two case reports and review of the literature. Am J Perinatol 2000;17(03):151-158

11 Saxonhouse MA. Management of neonatal thrombosis. Clin Perinatol 2012;39(01):191-208

12 Giacoia GP. High-dose urokinase therapy in newborn infants with major vessel thrombosis. Clin Pediatr (Phila) 1993;32(04): 231-237

13 Diaz R, Moffett BS, Karabinas S, Guffrey D, Mahoney DH Jr, Yee DL. Antithrombin concentrate use in children receiving unfractionated heparin for acute thrombosis. J Pediatr 2015;167(03): 645-649

14 Green PH. On a new variety of the femoral artery: with observations. Lancet 1832;17:730-731

15 van Hooft IM, Zeebregts CJ, van Sterkenburg SM, de Vries WR, Reijnen MM. The persistent sciatic artery. Eur J Vasc Endovasc Surg 2009;37(05):585-591

16 Shah SK, Phan NB, Doshi S, Richardson CJ. Symptomatic persistent sciatic artery in a newborn. J Pediatr Surg 2008;43(09): $1741-1744$ 